\title{
Painting with X-rays: virtual beams for ideal data collection
}

\author{
James M Holton ${ }^{a, b, c}$ \\ a Department of Biochemistry and Biophysics, University of California San Francisco \\ ${ }^{b}$ Division of Molecular Biophysics and Integrated Bioimaging, Lawrence Berkeley National Lab \\ ${ }^{c}$ Structural Molecular Biology Group, Stanford Synchrotron Radiation Lab, SLAC \\ JMHolton@lbl.gov
}

Radiation damage studies are almost always hampered by the uncertainty introduced by dosecontrast effects, and this becomes increasingly difficult with micro-beams. At extreme exposure the radiation damage reaction can even appear to run backwards as heavily-dosed regions burn out and the weak outskirts of the beam are all that is left still contributing to spots. RADDOSE3D [1-3] was created to address these effects, but what if you want to do the "control experiment" and have every cubic micron of illuminated crystal exposed to the same dose instead of a range? One way to do this is to raster the beam over an area much wider than the data-collection beam. As long as the dose deposited by the rotation data is small when compared to the raster dose, you can reliably get a nice, flat dose profile and a steady progression of dose from dataset to dataset. This, however, becomes impractical as the crystal gets smaller and smaller and the dose required to get a dataset becomes a significant fraction of the half-dose of the reaction of interest. For these cases interspersing "clean-up" data sets that partially overlap the main illuminated area can be very effective at flattening the final dose profile. For example, when shooting a thin needle crystal illuminated by a Gaussian beam with full-width at half-max (FWHM) 100 microns, placing three spots in a row spaced every 78.2 microns makes the dose contrast flat to within $0.1 \%$ across the central 100 microns of crystal length. All that is required is that these three spots be visited in round-robin fashion as the data are collected to prevent any one of them from getting too far ahead of the others. Similar "cleanup" geometries can be used for different crystal shapes, but in general spacing Gaussian beam spots at roughly one FWHM apart results in satisfyingly flat dose profiles.

[1] O.B. Zeldin, S. Brockhauser, J. Bremridge, J.M. Holton, E.F. Garman, Predicting the X-ray lifetime of protein crystals, PNAS USA, 110 (2013) 20551-20556.

[2] O.B. Zeldin, M. Gerstel, E.F. Garman, RADDOSE-3D: time- and space-resolved modelling of dose in macromolecular crystallography, J. Appl. Cryst., 46 (2013) 1225-1230.

[3] C.S. Bury, J.C. Brooks-Bartlett, S.P. Walsh, E.F. Garman, Estimate your dose: RADDOSE-3D, Protein Sci., 27 (2018) 217-228. 